\title{
KMT2A-rearranged diffuse large B-cell lymphoma in a child: a case report and molecular characterization
}

\author{
Shota Kato ${ }^{1}$, Yasuo Kubota ${ }^{1}$, Masahiro Sekiguchi ${ }^{1}$, Kentaro Watanabe ${ }^{1}$, Aya \\ Shinozaki-Ushiku ${ }^{1}$, Junko Takita ${ }^{1}$, and Mitsuteru Hiwatari ${ }^{1}$ \\ ${ }^{1}$ The University of Tokyo
}

July 1, 2020

\begin{abstract}
A 19-month-old female with a right temporal bone tumor was diagnosed with diffuse large B-cell lymphoma by tumor biopsy. Leukemic conversion occurred after chemotherapy for mature B-cell lymphoma. Additional chemotherapy was unsuccessful and resulted in death of the patient owing to disease progression. We detected the KMT2A-MLLT3 fused transcript in the bone marrow of the patient with primary and recurrent cancer. RNA sequencing of the bone marrow with recurrent cancer showed similarities with KMT2A-rearranged leukemia, such as overexpression of MEIS1 and MEF2C, which was attributable to the aggressive course.
\end{abstract}

\section{INTRODUCTION}

Diffuse large B-cell lymphoma (DLBCL) is the most common type of non-Hodgkin lymphoma in adults, while it is rare in children. DLBCL is a heterogeneous subtype of mature B-cell lymphoma characterized by the diffuse proliferation of B-cell antigen-expressing lymphoma cells larger than histiocyte nuclei. DLBCL is associated with frequent gene translocations, such as IGH -BCL6, IGH-BCL2, and IGH -MYC. ${ }^{1}$

KMT2A rearrangement is associated with leukemogenesis in acute lymphoblastic leukemia (ALL) and acute myeloid leukemia. KMT2Arearrangement in acute leukemia accounts for $5-10 \%$ of the total cases and correlates with poor prognosis. ${ }^{2}$ However, KMT2A -rearranged lymphoma has not been studied in detail.KMT2A -rearranged lymphomas, especially KMT2A-rearranged DLBCL, are less frequent than KMT2A -rearranged leukemia and the majority of those is constituted by lymphoblastic lymphoma. ${ }^{3-8}$ KMT2A -rearranged DLBCL can be detected in a few adult and pediatric patients. ${ }^{9,10}$

Herein, we describe a pediatric case of KMT2A -rearranged DLBCL. We performed RNA sequencing (RNAseq) to understand the molecular biology of KMT2A -rearranged DLBCL.

\section{METHODS}

This study was approved by the Human Genome, Gene Analysis Ethics Committee of the University of Tokyo (approval number G0948). Bone marrow (BM) samples collected were analyzed after obtaining written informed consent from legal guardians of the patient.

Total RNA was extracted from recurrent BM and checked for integrity and concentration using the Agilent Tapestation (Agilent, Santa Clara, CA, USA). RNA-seq libraries were constructed using the NEBNext Ultra RNA library prep kit compatible with the Illumina platform (New England BioLabs, Ipswich, MA, USA). Next-generation sequencing was performed using the Illumina NovaSeq 6000 platform with a standard $101 \mathrm{bp}$ paired-end read protocol according to the manufacturer's instructions. Reference genome (hg19) alignment and fusion gene detection were performed using Genomon v2.6.2. For expression analysis, fragments per kilobase of exon per million reads mapped (FPKM) were used as normalized count data. T-distributed 
stochastic neighbor embedding (t-SNE) was performed using R (v3.6.1) package Rtsne to reduce dimensions. For t-SNE, RNA-seq data of DLBCL and KMT2A -rearranged ALL were obtained from published studies (accession number GSE147986 from Gene Expression Omnibus [ncbi.nlm.nih.gov/geo] for DLBCL and a personal communication for KMT2A -rearranged ALL). ${ }^{11,12}$

Primary and recurrent BM samples were subjected to reverse transcription polymerase chain reaction (RTPCR) and Sanger sequencing ofKMT2A - MLLT3 and internal tandem duplication (ITD) and variants in N676 and D835 of FLT3. ITD and the variants ofFLT3 are additional gene alterations in KMT2A rearranged leukemia. ${ }^{13,14}$ Supplemental Table S1 lists the primer sequences.

\section{RESULTS}

A 19-month-old female with right facial nerve palsy was referred to the University of Tokyo Hospital. She had no personal or family history of cancer. Magnetic resonance imaging revealed a tumor lesion that filled the right temporal bone (Fig. 1A). Enhanced computed tomography showed bilateral renal involvement (Fig. 1B). Lymph nodes and the thymus were intact. Biopsy of the tumor tissue revealed DLBCL consisting of diffuse proliferation of centroblasts (Figs. 1C-F). The tumor infiltrated the central nervous system and was diagnosed as Murphy stage IV DLBCL. BM samples aspirated from the bilateral ilia showed normal karyotype and no morphological evidence of tumor invasion.

She was administered short-pulse intensive chemotherapy to treat mature B-cell lymphoma. ${ }^{15}$ Cytology of the cerebrospinal fluid was normal one month after initiating chemotherapy. The tumor nearly dissolved after chemotherapy, except for a small residual lesion in the right mastoid observed by gadolinium enhancement magnetic resonance imaging. Although discharged seven months after diagnosis, leukocytosis with blast cells in peripheral blood developed 2 months after completion of the chemotherapy. Flow cytometry showed abnormal lymphocytes with characteristics of the mature B-lineage $\left(\mathrm{CD} 10^{+}, \mathrm{CD} 19^{+}\right.$, and $\mathrm{CD} 20^{+}$, partially positive for immunoglobulin light chain lambda, and CD34-) in the BM. G-banding analysis and fluorescence in situ hybridization showed addition of 11q23 and KMT2A rearrangement, respectively. RT-PCR confirmed the presence of the KMT2A -MLLT3 fusion not only in sample at the recurrent timepoint, but also in that upon initial presentation, which demonstrated normal morphology and karyotype. Since leukocytosis worsened after reinitiating ALL-specific chemotherapy, ${ }^{16}$ she underwent hematopoietic cell transplantation during non-remission that resulted in the expansion of blast cells after neutrophil engraftment. She continued palliative chemotherapy with bortezomib. Unfortunately, the disease progressed and she died 18 months after diagnosis.

RNA-seq on BM during recurrent cancer identified theKMT2A -MLLT3 fusion including exons 1-9 and 6-11 of KMT2A and MLLT3, respectively, which was validated by Sanger sequencing (Figs. 2A-B). Other fusions, such as $I G H-B C L 6, I G H-B C L 2$, and $I G H-M Y C$, were not detected and no mutations were found inKRAS , NRAS , PTPN11, and FLT3 . ITD and the N676 and D835 variants of FLT3 were not identified by RT-PCR and Sanger sequencing of the primary or recurrent BM.

MEIS1 and MEF2C, which are highly expressed in KMT2A -rearranged leukemia, ${ }^{17}$ were overexpressed in the BM during cancer recurrence (60 and $83 \mathrm{FPKM}$, respectively); however, expression of posterior HOXA genes (HOXA6 ,7 , 9, and 10 ), also upregulated inKMT2A -rearranged leukemia, were not detected. IGLL5levels, involved in the expression of IGLJ1 and IGLC1, were elevated (400 FPKM), whereas the expression of $C D 34$ and DNTT (encoding terminal deoxynucleotidyl transferase) was not elevated (0.11 and 0.80 FPKM, respectively). t-SNE plots showed that our patient formed a part of the KMT2A -rearranged ALL, but not DLBCL, cluster (Fig. 2C).

\section{DISCUSSION}

We have described an intractable case of pediatric DLBCL with KMT2A rearrangement. The patient showed histological similarities with DLBCL; however, she exhibited disease relapse with leukemic conversion soon after chemotherapy against mature B-cell lymphoma. She was resistant to the salvage chemotherapy.

The KMT2A -MLLT3 fusion in BM motivated us to further investigate the biological features of the cancer. 
RNA-seq did not identify other fusions associated with DLBCL, but revealed the overexpression of genes associated with KMT2A -rearranged leukemia, such as MEIS1.${ }^{17}$ Moreover, the patient formed a part of the KMT2A -rearranged ALL cluster in the t-SNE plot. These results suggested that KMT2A -rearranged DLBCL shares biological features with KMT2A -rearranged leukemia, thereby resulting in resistance to treatment.

In contrast, the posterior HOXA cluster, upregulated inKMT2A -rearranged leukemia, was not overexpressed. ${ }^{17}$ Posterior HOXA is maximally expressed at the progenitor stage ${ }^{18}$ therefore, the low expression of posterior $H O X A$ in our patient could be attributed to the maturity of lymphoma cells (confirmed by flow cytometry and RNA-seq). Although KMT2A rearrangement is assumed to occur in early B-cell precursors, additional genetic aberrations, such as mutations in RAS pathway genes or tyrosine kinase domain mutations or ITD in FLT3, are pivotal for leukemogenesis. ${ }^{13,14,19,20}$ KMT2A -rearranged B-lineage ALL mainly includes pro-B-ALL, the most immature phenotype of precursor B-cell ALL; ${ }^{19}$ whereas someKMT2A -rearranged lymphoblastic lymphoma has a mature B-cell phenotype. ${ }^{4,6}$ Thus, the stage of differentiation in which aberrations other than KMT2A rearrangement occur might determine disease phenotype, i.e., development of ALL or lymphoma.

KMT2A -rearranged DLBCL has been reported in two adults and one child. ${ }^{9,10}$ The adults maintained remission after R-CHOP chemotherapy (cyclophosphamide, doxorubicin, vincristine, prednisolone, and rituximab). ${ }^{9}$ The pediatric case demonstrated poor response to mature B-cell lymphoma-specific chemotherapy and was in remission after high-dose cytarabine and oral maintenance chemotherapy with 6 -mercaptopurine and methotrexate. ${ }^{10}$ This difference in therapeutic response between the adult and pediatric cases, including our patient, may be attributed to differences in their genetic background. However, previous reports have not identified genetic alterations other than KMT2A rearrangement.

In conclusion, although further investigation is warranted, there might be similarities between the characteristics of KMT2A -rearranged DLBCL and leukemia at the molecular level.

\section{Acknowledgements:}

We acknowledge M. Matsumura and F. Saito for their technical assistance and Editage (www.editage.jp) for the English language review.

\section{Conflict of interest:}

The authors declare no conflict of interest.

\section{References}

1. Li S, Young KH, Medeiros LJ. Diffuse large B-cell lymphoma.Pathology. 2018;50(1):74-87.

2. Yokoyama A. Molecular mechanisms of MLL-associated leukemia.Int J Hematol. 2015;101(4):352-361.

3. Shafer D, Wu H, Al-Saleem T, et al. Cutaneous precursor B-cell lymphoblastic lymphoma in 2 adult patients: clinicopathologic and molecular cytogenetic studies with a review of the literature.Arch Dermatol. 2008;144(9):1155-1162.

4. Takachi T, Iwabuchi H, Imamura M, Imai C. Lymphoblastic lymphoma with mature B-cell immunophenotype and MLL-AF9 in a child. Pediatr Blood Cancer. 2011;57(7):1251-1252.

5. Quigg TC, Haddad NG, Buchsbaum JC, Shih CS. Hypothalamic obesity syndrome: rare presentation of CNS+ B-cell lymphoblastic lymphoma.Pediatr Blood Cancer. 2012;59(5):930-933.

6. Ahlmann M, Meyer C, Marschalek R, et al. Complex MLL rearrangement in non-infiltrated bone marrow in an infant with stage II precursor B-lymphoblastic lymphoma. Eur J Haematol. 2014;93(4):349-353.

7. Larish AM, Dolan M, Casey T, Perkins JL. Bilateral ovarian B-lineage lymphoblastic lymphoma with MLL gene rearrangement: a novel case in infancy. J Pediatr Hematol Oncol. 2015;37(4):e215-217. 
8. Thandla S, Alashari M, Green DM, Aplan PD. Therapy-related T cell lymphoblastic lymphoma with t(11;19)(q23;p13) and MLL gene rearrangement. Leukemia. 1999;13(12):2116-2118.

9. Gindin T, Murty V, Alobeid B, Bhagat G. MLL/KMT2A translocations in diffuse large B-cell lymphomas. Hematol Oncol.2015;33(4):239-246.

10. Hiroki H, Yoshimi A, Kato K, Yanai T, Koike K. Primary Cutaneous Diffuse Large B-Cell Lymphoma With KMT2A-MLLT3: An Infantile Case Study.J Pediatr Hematol Oncol. 2019;41(8):657-658.

11. Yan W, Jiang X, Wang W, et al. Cell-of-Origin Subtyping of Diffuse Large B-Cell Lymphoma by Using a qPCR-based Gene Expression Assay on Formalin-Fixed Paraffin-Embedded Tissues. Front Oncol.2020;10:803.

12. Hirabayashi S, Ohki K, Nakabayashi K, et al. ZNF384-related fusion genes define a subgroup of childhood B-cell precursor acute lymphoblastic leukemia with a characteristic immunotype.Haematologica. 2017;102(1):118-129.

13. Hyrenius-Wittsten A, Pilheden M, Sturesson H, et al. De novo activating mutations drive clonal evolution and enhance clonal fitness in KMT2A-rearranged leukemia. Nat Commun. 2018;9(1):1770.

14. Tsutsumi S, Taketani T, Nishimura K, et al. Two distinct gene expression signatures in pediatric acute lymphoblastic leukemia with MLL rearrangements. Cancer Res. 2003;63(16):4882-4887.

15. Tsurusawa M, Mori T, Kikuchi A, et al. Improved treatment results of children with B-cell nonHodgkin lymphoma: a report from the Japanese Pediatric Leukemia/Lymphoma Study Group B-NHL03 study. Pediatr Blood Cancer. 2014;61(7):1215-1221.

16. Takahashi H, Kajiwara R, Kato M, et al. Treatment outcome of children with acute lymphoblastic leukemia: the Tokyo Children's Cancer Study Group (TCCSG) Study L04-16. Int J Hematol.2018;108(1):98108.

17. Krivtsov AV, Twomey D, Feng Z, et al. Transformation from committed progenitor to leukaemia stem cell initiated by MLL-AF9. Nature.2006;442(7104):818-822.

18. Forsberg EC, Prohaska SS, Katzman S, Heffner GC, Stuart JM, Weissman IL. Differential expression of novel potential regulators in hematopoietic stem cells. PLoS Genet. 2005;1(3):e28.

19. Jansen MW, Corral L, van der Velden VH, et al. Immunobiological diversity in infant acute lymphoblastic leukemia is related to the occurrence and type of MLL gene rearrangement. Leukemia.2007;21(4):633-641.

20. Andersson AK, Ma J, Wang J, et al. The landscape of somatic mutations in infant MLL-rearranged acute lymphoblastic leukemias. Nat Genet. 2015;47(4):330-337.

\section{Legends}

FIGURE 1 A, Magnetic resonance T2-weighted imaging of the head. The tumor occupied the right external acoustic meatus, tympanic cavity, mastoid cells, and internal acoustic meatus with invasion into the right temporal muscle (arrow). B, Contrast-enhanced abdominal computed tomography. The kidneys were bilaterally enlarged with poorly enhanced lesions. C-F, Histopathologic findings of the tumor tissue. C, Hematoxylin and eosin staining. Infiltration of medium- to large-sized centroblasts. D, Immunohistochemical staining for MIB-1. The MIB-1 proliferation index was 80-90\%. E and F, Immunohistochemical staining for CD20 (E) and BCL2 (F). The centroblasts were positive for CD10, CD20, CD79a, and BCL2 and negative for CD3, CD5, terminal deoxynucleotidyl transferase, and Epstein-Barr virus-encoded small RNAin situ hybridization.

FIGURE 2 A, DNA, mRNA, and protein conformation of theKMT2A -MLLT3 fusion. MBM, MENINbinding motif; IBM, integrase domain-binding motif; zf, zinc finger; AHD, ANC1 homology domain. B, Sanger sequencing showing the fusion between exon 9 ofKMT2A and exon 6 of MLLT3 . C, t-SNE plot 
generated using the RNA-seq data from the patient and patients with DLBCL and KMT2A -rearranged ALL obtained from published studies. ${ }^{11,12}$

SUPPLEMENTAL TABLE S1 Primer sequences for reverse transcription-polymerase chain reaction.

\section{Hosted file}

figure_1.pdf available at https://authorea.com/users/338613/articles/465108-kmt2a-rearrangeddiffuse-large-b-cell-lymphoma-in-a-child-a-case-report-and-molecular-characterization

A

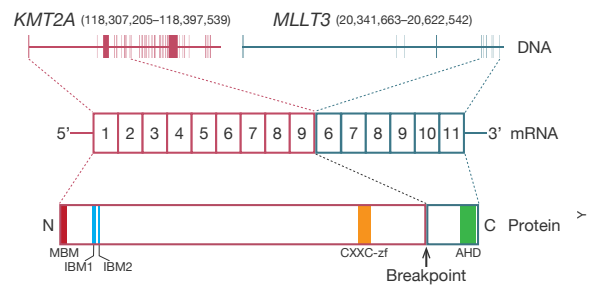

B

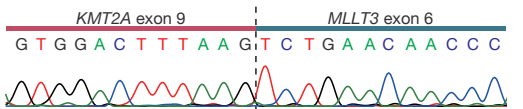

C

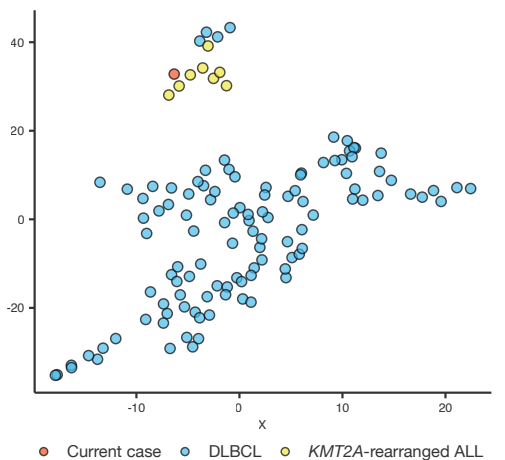

